\title{
Mathematical and numerical modeling of plate dynamics with rotational inertia
}

\author{
Francesco Bonaldi, Giuseppe Geymonat, Françoise Krasucki and \\ Marina Vidrascu
}

\begin{abstract}
We give a presentation of the mathematical and numerical treatment of plate dynamics problems including rotational inertia. The presence of rotational inertia in the equation of motion makes the study of such problems interesting. We employ HCT finite elements for space discretization and the Newmark method for time discretization in FreeFEM++, and test such methods in some significant cases: a circular plate clamped all over its lateral surface, a rectangular plate simply supported all over its lateral surface, and an $L$-shaped clamped plate.
\end{abstract}

Keywords. Plates, Kirchhoff-Love, Rotational inertia, FreeFEM, HCT, Newmark.

2010 Mathematics Subject Classification. 74K20, 65M60, 35Q74.

\section{General notation}

We denote by $\Omega \subset \mathbb{R}^{2}$ a two-dimensional domain with smooth boundary $\Gamma$; $\Gamma_{0} \subset \Gamma$ is a measurable subset of $\Gamma$, with strictly positive length measure, and $\Gamma_{1}=\Gamma \backslash \Gamma_{0}$. The outer unit normal and tangent vector fields on $\Gamma$ are denoted, respectively, by $\mathbf{n}=\left(n_{1}, n_{2}\right)$ and $\boldsymbol{\tau}=\left(-n_{2}, n_{1}\right)$. For $\psi$ a real-valued field defined on $\bar{\Omega}, \partial_{n} \psi=\nabla \psi \cdot \mathbf{n}$ and $\partial_{\tau} \psi=\nabla \psi \cdot \boldsymbol{\tau}$ denote its normal and tangential derivatives on $\Gamma$. The space dependence of a field is left tacit, unless noted otherwise. The time derivative of a real- or vector-valued field $\varphi$ is denoted by $\dot{\varphi}$, of a function $\Phi$ taking values in a Hilbert space by $\frac{\mathrm{d} \Phi}{\mathrm{d} t}$. Finally, $\operatorname{Sym}(2)$ denotes the space of symmetric second-order tensors in $\mathbb{R}^{2}$.

\section{Introduction}

In [6] the equations of plate models for magneto-electro-thermo-elastic sensors and actuators have been deduced by an asymptotic development with respect to

This work has been partially supported by the French Agence Nationale de la Recherche (ANR) under grant ARAMIS (Projet «Blanc», N. ANR 12 BS01-0021) (Analysis of Robust Asymptotic Methods in Numerical Simulation in Mechanics). 
the thickness $2 e>0$ of the plate. A peculiar feature of the different models concerns the flexural problem, governing the evolution of the transversal displacement $w$ of the plate, which occupies the closure of the three-dimensional domain $\Omega \times(-e, e)$ in its reference configuration and whose kinematics is of Kirchhoff-Love type. This problem is uncoupled from the membrane problem, it takes into account an inertia effect involving the mean curvature of the deformed middle surface, referred to as rotational inertia, and the only influence of magneto-electro-thermo-elastic behavior of the material appears in the coefficients $\mathbb{A}=\left(A_{\alpha \beta \sigma \tau}\right)$ of the (symmetric) moment tensor $\mathbf{M}(t)=\left(M_{\alpha \beta}(t)\right)=$ $-\frac{2 e^{3}}{3} \mathbb{A} \nabla \nabla w(t), \quad(\nabla \nabla w(t))_{\alpha \beta}=\partial_{\alpha \beta} w(t)$ (let us point out that the fourthorder tensor $\mathbb{A}=\left(A_{\alpha \beta \sigma \tau}\right)$ is symmetric and strongly elliptic). The transverse displacement $w$ of the plate is solution of the following evolution equation:

$$
2 e \rho \ddot{w}-\frac{2 e^{3}}{3} \rho \triangle \ddot{w}-\operatorname{div} \operatorname{div} \mathbf{M}=f+\operatorname{div} \mathbf{m} \quad \text { in } \Omega \times(0, T),
$$

equipped with initial conditions

$$
w(0)=w_{0}, \dot{w}(0)=w_{1} \quad \text { in } \Omega
$$

and with suitable boundary conditions, where $\rho>0$ is the mass density of the plate, supposed to be homogeneous. If one supposes, for the sake of simplicity, that the three-dimensional body is subjected to time-dependent volume forces $(0,0, \mathcal{F}(t))$ and surface loads $\left(\mathcal{G}_{1}^{ \pm}(t), \mathcal{G}_{2}^{ \pm}(t), 0\right)$ on the upper and lower faces $\Omega \times\{ \pm e\}$, then the source terms in the right-hand side of (1) have the following expressions

$$
f(t)=\int_{-e}^{+e} \mathcal{F}(t) \mathrm{d} x_{3}, \quad m_{\alpha}(t)=e\left(\mathcal{G}_{\alpha}^{+}(t)-\mathcal{G}_{\alpha}^{-}(t)\right), \quad \alpha \in\{1,2\} .
$$

The term accounting for rotational inertia in (1) is $-\frac{2 e^{3}}{3} \rho \triangle \ddot{w}$, and it derives from the expression of the volume kinetic energy $k(t)$ for Kirchhoff-Love plates subjected to pure bending:

$$
k(t)=\frac{1}{2}\left(2 e \rho \dot{w}^{2}+\frac{2 e^{3}}{3} \rho|\nabla \dot{w}|^{2}\right) .
$$

The reason for the word rotational is that the quantity $|\nabla w|$ gives a measure of the rotation of the material fibers orthogonal to the middle surface of the plate, which remain straight and perpendicular to this surface after deformation in KirchhoffLove kinematics. 
The influence of rotational inertia on the lateral vibrations of linearly elastic bars was considered by Lord Rayleigh [20], sect. 186; the extension of this analysis to the flexural motion of isotropic elastic plates was carried out for the first time in 1951 in a seminal paper by R. D. Mindlin [16] where also the effect of transverse shear deformation is taken into account. Later on, an evolution model for plates with rotational inertia was deduced by A. Raoult [18] using the asymptotic expansion method. The influence of such effects has also been considered in the case of flexural motion of large amplitude. Analogously to the case of beams, where it can be worth to account for rotational inertia of the cross section in case the slenderness of the beam (i.e., the ratio of the cross section diameter to the length of the beam) is lower than one, but not very close to zero, in our case rotational inertia of the material fibers orthogonal to the middle surface may be important if the plate thinness (i.e., the ratio of the thickness to the diameter of the plate) is lower than one, but not very close to zero. In the following, to point out the influence of the thickness parameter, we carry out our analysis on equation (1) divided by $2 e$.

Our goal, after providing a result of well-posedness for the problem, is to test a numerical method involving $C^{1}$ elements for what concerns space discretization and Newmark's midpoint method concerning time discretization. The presence of the rotational inertia requires an appropriate choice of the functional spaces, presented in sect. 1, where we sketch the proof of the well-posedness using the Faedo-Galerkin method. In sect. 2 we perform, in the appropriate Sobolev spaces, the error analysis of a finite element spatial discretization and of a Newmark-type discrete time approximation. Our choice allows the application of the methods developed for linear second-order evolution equations. Based on a continuoustime Galerkin method (see e.g. [2] or [11]) we can infer optimal error estimates, and then couple with the error estimates for the time discretization of Newmark type (see e.g. [19]). Note that a conforming space discretization for plates requires $C^{1}$ elements; we choose HCT elements (see e.g. [7]). This theoretical numerical analysis is complemented with numerical tests performed with FreeFEM++.

Let us remark that the choice of $C^{1}$ elements is not very common in practice, as these elements are rather expensive. Mixed and hybrid plate elements were extensively studied. Among the elements widely used in the engineering community we can mention the DKT triangle [3]. For a general review of plate elements see for example [5], [14] and references therein. We can also mention the more modern hybrid high-order methods [8,9]: they will be studied in a forthcoming paper. 


\section{Existence and uniqueness}

We start by rewriting (1) as

$$
\rho \ddot{w}-\frac{e^{2}}{3} \rho \Delta \ddot{w}-\frac{e^{2}}{3} \operatorname{div} \operatorname{div} \mathbb{A} \nabla \nabla w=\frac{1}{2 e}(f+\operatorname{div} \mathbf{m}) .
$$

The weak formulation of the problem depends on boundary conditions. For this, we first define the pivot space

$$
H=\left\{u \in H^{1}(\Omega): u=0 \text { on } \Gamma_{0}\right\}
$$

and the bilinear form $b: H \times H \rightarrow \mathbb{R}$ given by

$$
b(u, v)=\int_{\Omega}\left(\rho u v+\frac{e^{2}}{3} \rho \nabla u \cdot \nabla v\right) \mathrm{d} \Omega, \quad \forall u, v \in H .
$$

Notice that $b(\cdot, \cdot)$ defines a scalar product in $H$ whose associated norm $|\cdot|_{b}$ is equivalent to the usual Sobolev norm.

Let $V$ be a Hilbert-Sobolev space such that

(i) $V \subseteq H^{2}(\Omega) \cap H$,

(ii) the embedding $V \hookrightarrow H$ is compact.

Thanks to $(i)$, we can endow $V$ with the Hessian $L^{2}$-norm given by

$$
\|v\|=\left(\int_{\Omega} \nabla \nabla v: \nabla \nabla v \mathrm{~d} \Omega\right)^{1 / 2}
$$

Let us define the bilinear form $a: V \times V \rightarrow \mathbb{R}$ by

$$
a(u, v)=\frac{e^{2}}{3} \int_{\Omega} \mathbb{A} \nabla \nabla u: \nabla \nabla v \mathrm{~d} \Omega, \quad \forall u, v \in V .
$$

By the symmetry and ellipticity properties of $\mathbb{A}$, there exist two positive constants $\mathcal{A}^{-}$and $\mathcal{A}^{+}$such that, for all $x \in \Omega$,

$$
\mathcal{A}^{-}|\mathbf{U}|^{2} \leqslant \mathbb{A} \mathbf{U}: \mathbf{U} \leqslant \mathcal{A}^{+}|\mathbf{U}|^{2}, \quad \forall \mathbf{U} \in \operatorname{Sym}(2) .
$$

Hence, $a(\cdot, \cdot)$ is symmetric and $V$-elliptic: $a(v, v) \geqslant \frac{e^{2}}{3} \mathcal{A}^{-}\|v\|^{2}$ for any $v \in V$. In the numerical examples, we consider essentially the following situations:

$$
(B C)_{1}: \begin{cases}\frac{2 e^{3}}{3} \rho \partial_{n} \ddot{w}+\operatorname{div} \mathbf{M} \cdot \mathbf{n}+\partial_{\tau}(\mathbf{M n} \cdot \boldsymbol{\tau})=-\mathbf{m} \cdot \mathbf{n} & \text { on } \Gamma_{1} \times(0, T), \\ \mathbf{M n} \cdot \mathbf{n}=0 & \text { on } \Gamma_{1} \times(0, T), \\ w=0, \partial_{n} w=0 & \text { on } \Gamma_{0} \times(0, T)\end{cases}
$$




$$
(B C)_{2}: \begin{cases}\frac{2 e^{3}}{3} \rho \partial_{n} \ddot{w}+\operatorname{div} \mathbf{M} \cdot \mathbf{n}+\partial_{\tau}(\mathbf{M n} \cdot \boldsymbol{\tau})=-\mathbf{m} \cdot \mathbf{n} & \text { on } \Gamma_{1} \times(0, T), \\ \mathbf{M n} \cdot \mathbf{n}=0 & \text { on } \partial \Omega \times(0, T), \\ w=0 & \text { on } \Gamma_{0} \times(0, T) .\end{cases}
$$

Boundary conditions $(B C)_{1}$ refer to a plate clamped on $\Gamma_{0}$; in this case we choose

$$
V=V_{1}=\left\{u \in H^{2}(\Omega): u=\partial_{n} u=0 \text { on } \Gamma_{0}\right\} .
$$

Boundary conditions $(B C)_{2}$ feature a plate simply supported on $\Gamma_{0}$, in which case we choose

$$
V=V_{2}=\left\{u \in H^{2}(\Omega): u=0 \text { on } \Gamma_{0}\right\}=H^{2}(\Omega) \cap H .
$$

In both cases, under the general hypothesis of anisotropic linearly elastic behavior, the weak formulation of the problem has the following aspect:

$$
\left\{\begin{array}{l}
\text { For any fixed } t \in(0, T), \text { find } w(t) \in V \text { such that } \\
\int_{\Omega}\left(\rho \ddot{w}(t) v+\frac{e^{2}}{3} \rho \nabla \ddot{w}(t) \cdot \nabla v+\frac{e^{2}}{3} \mathbb{A} \nabla \nabla w(t): \nabla \nabla v\right) \mathrm{d} \Omega \\
=\frac{1}{2 e} \int_{\Omega}(f(t) v-\mathbf{m}(t) \cdot \nabla v) \mathrm{d} \Omega,
\end{array}\right.
$$

where $V=V_{1}$ or $V=V_{2}$.

Remark 1.1. Notice that, by taking volume and surface loads constant in space in (3), we have $f(t)=2 e \mathcal{F}(t)$ and $m_{\alpha}(t)=e\left(\mathcal{G}_{\alpha}^{+}(t)-\mathcal{G}_{\alpha}^{-}(t)\right)$, so that the right-hand sides of (4) and of (11) are independent of the thickness.

In order to show that problem (11) is well-posed, we identify the time-dependent linear form on $H$

$$
L_{t}(v)=\frac{1}{2 e} \int_{\Omega}(f(t) v-\mathbf{m}(t) \cdot \nabla v) \mathrm{d} \Omega,
$$

with the scalar product of an element $F(t) \in H$ (for $0<t<T$ ) with $v \in H$. This can be accomplished via the following problem:

$$
\begin{gathered}
\text { Find } F(t) \in H \text { such that } \\
b(F(t), v)=L_{t}(v), \quad \forall v \in H .
\end{gathered}
$$

Provided $f(t) \in L^{2}(\Omega)$ and $\mathbf{m}(t) \in \mathbf{L}^{2}(\Omega)$ for $0<t<T$, problem (12) is well-posed by the Lax-Milgram Lemma. As for problem (11), we assume 
$f \in L^{2}\left(0, T ; L^{2}(\Omega)\right)$ and $\mathbf{m} \in L^{2}\left(0, T ; \mathbf{L}^{2}(\Omega)\right)$, so that $F \in L^{2}(0, T ; H)$; the formulation of the problem reads then:

Given $w_{0} \in V, w_{1} \in H$ and $F \in L^{2}(0, T ; H)$, find a function $w$ such that

$$
\begin{gathered}
w \in C^{0}([0, T] ; V) \cap C^{1}([0, T] ; H) \text { and, for all } v \in V, \\
\frac{\mathrm{d}^{2}}{\mathrm{~d} t^{2}} b(w(t), v)+a(w(t), v)=b(F(t), v), \\
w(0)=w_{0}, \frac{\mathrm{d} w}{\mathrm{~d} t}(0)=w_{1} .
\end{gathered}
$$

Theorem 1.2. Let $T>0$ be fixed, $w_{0} \in V, w_{1} \in H$ and $F \in L^{2}(0, T ; H)$.

(i) There exists a unique function $w \in C^{0}([0, T] ; V) \cap C^{1}([0, T] ; H)$ satisfying (13).

(ii) For all $t \in[0, T]$, the function $w$ satisfies the following energy equation:

$$
|\dot{w}(t)|_{b}^{2}+a(w(t), w(t))=\left|w_{1}\right|_{b}^{2}+a(w(0), w(0))+2 \int_{0}^{t} b(F(s), \dot{w}(s)) \mathrm{d} s .
$$

(iii) There exists a constant $c=c(\Omega, \mathbb{A})$ such that

$$
\|w\|_{C^{0}([0, T] ; V)}+\|w\|_{C^{1}([0, T] ; H)} \leqslant \frac{\sqrt{T}}{e} c(\Omega, \mathbb{A})\left(\left\|w_{0}\right\|+\left|w_{1}\right|_{b}+\|F\|_{L^{2}(0, T ; H)}\right) .
$$

For a proof of statements (i) and (iii) of Theorem 1.2, of which we present hereinafter some essential parts, see e.g. [1] and [19] (see also [10], for a general treatment of evolution equations); the proof of statement (ii) can be found in [15, Chap. 3, Sect. 8] where a very general situation is considered. The uniqueness of the solution is proven in a standard way. With the choice (9) or (10) of the space $V$, and (5) for the space $H$, endowed with scalar products (8) and (6) respectively, the Faedo-Galerkin approximation method can be used. The compactness of the embedding $V \hookrightarrow H$ implies that there exists an increasing sequence of eigenvalues $0<\lambda_{1} \leqslant \lambda_{2} \leqslant \ldots \leqslant \lambda_{i} \leqslant \ldots$ and a Hilbert basis $\left\{g_{i}\right\}$, orthonormal in $H$ and orthogonal in $V$, of associated eigenvectors verifying

$$
\forall v \in V, \quad a\left(g_{i}, v\right)=\lambda_{i} b\left(g_{i}, v\right)
$$

(see e.g. [?]). As for existence, the subspace $V_{m}$ of $V$ generated by the first $m$ eigenvectors $g_{1}, \ldots, g_{m}$ is introduced; let $w_{m}: t \in[0, T] \mapsto w_{m}(t) \in V_{m}$ be solu- 
tion to the following (well-posed) second-order system of differential equations:

$$
\begin{gathered}
\forall v_{m} \in V_{m}, \quad \frac{\mathrm{d}^{2}}{\mathrm{~d} t^{2}} b\left(w_{m}(t), v\right)+a\left(w_{m}(t), v\right)=b(F(t), v), \\
w_{m}(0)=w_{0, m}=\sum_{i=1}^{m} b\left(w_{0}, g_{i}\right) g_{i}, \quad \frac{\mathrm{d} w_{m}}{\mathrm{~d} t}(0)=w_{1, m}=\sum_{i=1}^{m} b\left(w_{1}, g_{i}\right) g_{i} .
\end{gathered}
$$

One can show that $\left\{w_{m}\right\}_{m \in \mathbb{N}}$ is a Cauchy sequence in spaces $C^{0}([0, T] ; V)$ and $C^{1}([0, T] ; H)$ and thus it converges to a function $w$ in such spaces. Finally, by a density argument, it is proven that $w$ is solution to (13).

\section{Numerical analysis}

\subsection{Semi-discrete problem}

Let $V_{h} \subset V$ denote a subspace of $V$ of dimension $I=I(h)$ and consider the following semi-discrete problem: given $w_{0, h} \in V_{h}$ and $w_{1, h} \in V_{h}$, find the solution $w_{h}: t \in[0, T] \mapsto w_{h}(t) \in V_{h}$ to the following system of ordinary differential equations:

$$
\begin{gathered}
\forall v_{h} \in V_{h}, \quad \frac{\mathrm{d}^{2}}{\mathrm{~d} t^{2}} b\left(w_{h}(t), v_{h}\right)+a\left(w_{h}(t), v_{h}\right)=b\left(F(t), v_{h}\right), \\
w_{h}(0)=w_{0, h}, \frac{\mathrm{d} w_{h}}{\mathrm{~d} t}(0)=w_{1, h} .
\end{gathered}
$$

We now introduce a basis $\left\{\varphi_{i}\right\}_{1 \leqslant i \leqslant I}$ of $V_{h}$ and denote the time-dependent components of $w_{h}$ in this basis by $\xi_{j}(t), j=1, \ldots, I$. Analogously, we denote the components in the same basis of $w_{0, h}$ and $w_{1, h}$ respectively by $\xi_{0, j}$ and $\xi_{1, j}$. Finally, we set $\chi_{h j}(t)=b\left(F(t), \varphi_{j}\right)$. Then (16) reads

$$
\left\{\begin{array}{l}
\mathcal{M}_{h} \ddot{\boldsymbol{\xi}}(t)+\mathcal{K}_{h} \boldsymbol{\xi}(t)=\chi_{h}(t), \\
\boldsymbol{\xi}(0)=\boldsymbol{\xi}_{0}, \quad \dot{\boldsymbol{\xi}}(0)=\boldsymbol{\xi}_{1},
\end{array}\right.
$$

with self-explanatory notation. Matrices $\mathcal{M}_{h}$ and $\mathcal{K}_{h}$ are respectively the mass matrix and the stiffness matrix. Their coefficients are

$$
\left(\mathcal{M}_{h}\right)_{i j}=b\left(\varphi_{i}, \varphi_{j}\right) \quad \text { and } \quad\left(\mathcal{K}_{h}\right)_{i j}=a\left(\varphi_{i}, \varphi_{j}\right), \quad 1 \leqslant i, j \leqslant I .
$$

In the following, we drop the index $h$ when there is no ambiguity.

Remark 2.1. The choice of the scalar product (6) in $H$ ensures that $\mathcal{M}$ is positive definite. 
Problem (17) is the semi-discrete counterpart of (13). Under certain in-time regularity hypotheses on $w$ and adapting the arguments of [2], [11], it is possible to give an estimate of the error $w_{h}(t)-w(t)$. For this, we introduce the elliptic projection operator $\Pi_{h}$, a linear and continuous operator mapping $u \in V$ onto $\Pi_{h} u \in V_{h}$ defined by

$$
\forall v_{h} \in V_{h}, \quad a\left(\Pi_{h} u-u, v_{h}\right)=0 .
$$

We have the following results.

Theorem 2.2. Let $T>0$ be fixed and assume that the solution $w$ of (13) verifies $w \in C^{2}([0, T] ; V)$. Then there exists a constant $C=C(\mathbb{A})$ independent of $h$ such that, for any $t \in[0, T]$,

$$
\begin{aligned}
\left\|w_{h}(t)-w(t)\right\|+\mid \frac{\mathrm{d} w_{h}}{\mathrm{~d} t}(t) & -\left.\frac{\mathrm{d} w}{\mathrm{~d} t}(t)\right|_{b} \\
\leqslant \frac{1}{e} C(\mathbb{A})\left(\| w_{0, h}-\right. & \Pi_{h} w_{0}\left\|+\left|w_{1, h}-\Pi_{h} w_{1}\right|_{b}+\right\|\left(\mathbf{I}-\Pi_{h}\right) w(t) \| \\
& \left.+\left|\left(\mathbf{I}-\Pi_{h}\right) \frac{\mathrm{d} w}{\mathrm{~d} t}(t)\right|_{b}+\int_{0}^{t}\left|\left(\mathbf{I}-\Pi_{h}\right) \frac{\mathrm{d}^{2} w}{\mathrm{~d} t^{2}}(s)\right|_{b} \mathrm{~d} s\right) .
\end{aligned}
$$

Theorem 2.3. Under the assumptions of Theorem 2.2 let the following approximation hypotheses be satisfied:

$$
\begin{aligned}
\forall v \in V, \quad & \lim _{h \rightarrow 0} \inf _{v_{h} \in V_{h}}\left\|v-v_{h}\right\|=0 \\
& \lim _{h \rightarrow 0}\left\|w_{0, h}-w_{0}\right\|=0 \\
& \lim _{h \rightarrow 0}\left|w_{1, h}-w_{1}\right|_{b}=0
\end{aligned}
$$

then

$$
\forall t \in[0, T], \quad \lim _{h \rightarrow 0}\left(\left\|w_{h}(t)-w(t)\right\|+\left|\frac{\mathrm{d} w_{h}}{\mathrm{~d} t}(t)-\frac{\mathrm{d} w}{\mathrm{~d} t}(t)\right|_{b}\right)=0 .
$$

This convergence result can actually be improved; under the hypotheses of Theorem (1.2),

$$
\lim _{h \rightarrow 0} w_{h}=w \quad \text { in } C^{0}([0, T] ; V) \text { and in } C^{1}([0, T] ; H) .
$$




\subsection{Time discretization: Newmark's midpoint method}

A typical time discretization method widely used for differential systems of the form (17) is the Newmark method (see, e.g., [12]). In particular, we will use a midpoint approximation.

Let $N \in \mathbb{N}$; we introduce a time-step $\Delta t=\frac{T}{N}$ and a uniform mesh of the interval $[0, T]$ defined by nodes $t_{n}=n \Delta t, 0 \leqslant n \leqslant N$. We want to compute an approximation $\left(\boldsymbol{\xi}^{n}, \dot{\boldsymbol{\xi}}^{n}\right)$ of the pair $\left(\boldsymbol{\xi}\left(t_{n}\right), \dot{\boldsymbol{\xi}}\left(t_{n}\right)\right)$. The discretization of (17) based on Newmark's midpoint method can be rewritten as

$$
\left\{\begin{array}{l}
\left(\mathcal{M}+\frac{\Delta t^{2}}{4} \mathcal{K}\right) \boldsymbol{\xi}^{n+\frac{1}{2}}=\mathcal{M} \boldsymbol{\xi}^{n}+\frac{\Delta t}{2} \mathcal{M} \dot{\boldsymbol{\xi}}^{n}+\frac{\Delta t^{2}}{4} \chi^{n+\frac{1}{2}}, 0 \leqslant n \leqslant N-1, \\
\boldsymbol{\xi}^{0}=\boldsymbol{\xi}_{0}, \quad \dot{\boldsymbol{\xi}}^{0}=\boldsymbol{\xi}_{1}
\end{array}\right.
$$

with $\boldsymbol{\xi}^{n+\frac{1}{2}}=\frac{1}{2}\left(\boldsymbol{\xi}^{n+1}+\boldsymbol{\xi}^{n}\right)$ and $\chi^{n+\frac{1}{2}}=\frac{1}{2}\left(\chi\left(t_{n+1}\right)+\chi\left(t_{n}\right)\right)$. Upon solving (22) for $\xi^{n+\frac{1}{2}}$, the update rules are

$$
\begin{aligned}
\dot{\boldsymbol{\xi}}^{n+1} & =\frac{4}{\Delta t}\left(\boldsymbol{\xi}^{n+\frac{1}{2}}-\boldsymbol{\xi}^{n}\right)-\dot{\boldsymbol{\xi}}^{n}, \\
\boldsymbol{\xi}^{n+1} & =2 \boldsymbol{\xi}^{n+\frac{1}{2}}-\boldsymbol{\xi}^{n} .
\end{aligned}
$$

In this situation, Raviart and Thomas [19] have proved the following error estimate.

Theorem 2.4. Let $T>0$ be fixed. Then the solution $\left\{w_{h}^{n} \in V_{h}, 0 \leqslant n \leqslant N\right\}$ given by (22) verifies the following estimates:

(i) if $w \in C^{2}([0, T] ; V) \cap C^{3}([0, T] ; H)$,

$$
\begin{aligned}
& \left|w_{h}^{n}-w\left(t_{n}\right)\right|_{b} \leqslant C\left(\left|w_{0, h}-\Pi_{h} w_{0}\right|_{b}+\left|w_{1, h}-\Pi_{h} w_{1}\right|_{b}+\right. \\
& \left.+\left|\left(\mathbf{I}-\Pi_{h}\right) w\left(t_{n}\right)\right|_{b}+\int_{0}^{t_{n}}\left(\left|\left(\mathbf{I}-\Pi_{h}\right) \frac{\mathrm{d}^{2} w}{\mathrm{~d} t^{2}}(s)\right|_{b}+\Delta t\left|\frac{\mathrm{d}^{3} w}{\mathrm{~d} t^{3}}(s)\right|_{b}\right) \mathrm{d} s\right),
\end{aligned}
$$

where $C=C(T)$ is independent of $h, \Delta t$ and $w$;

(ii) if $w \in C^{2}([0, T] ; V) \cap C^{4}([0, T] ; H)$,

$$
\begin{aligned}
& \left|w_{h}^{n}-w\left(t_{n}\right)\right|_{b} \leqslant C\left(\left|w_{0, h}-\Pi_{h} w_{0}\right|_{b}+\left|w_{1, h}-\Pi_{h} w_{1}\right|_{b}+\right. \\
& \left.+\left|\left(\mathbf{I}-\Pi_{h}\right) w\left(t_{n}\right)\right|_{b}+\int_{0}^{t_{n}}\left(\left|\left(\mathbf{I}-\Pi_{h}\right) \frac{\mathrm{d}^{2} w}{\mathrm{~d} t^{2}}(s)\right|_{b}+\Delta t^{2}\left|\frac{\mathrm{d}^{4} w}{\mathrm{~d} t^{4}}(s)\right|_{b}\right) \mathrm{d} s\right),
\end{aligned}
$$

where $C=C(T)$ is independent of $h, \Delta t$ and $w$. 


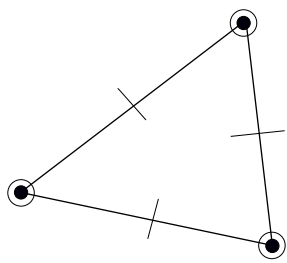

(a)

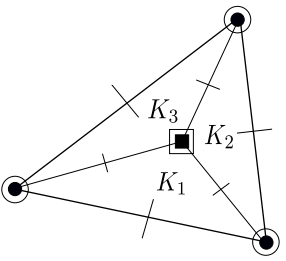

(b)

Figure 1. The HCT element (a) and the HCT element in detail (b).

\subsection{Space discretization: HCT elements}

The solution of (13) belongs to $C^{0}([0, T] ; V) \cap C^{1}([0, T] ; H)$, thus we are led to select finite elements of class $C^{1}$; in particular, we will use HCT elements.

Let $\mathcal{T}_{h}$ denote a regular mesh in the sense of Ciarlet [7,19] of the domain $\bar{\Omega}$, $K \in \mathcal{T}_{h}$ the typical element of $\mathcal{T}_{h}$ and $X_{h}$ a finite element space. Moreover, let $P_{K}=\left\{v_{h \mid K}: v_{h} \in X_{h}\right\}$. We recall the following result of [7].

Theorem 2.5. Assume that the inclusions $P_{K} \subset H^{2}(K)$ for all $K \in \mathcal{T}_{h}$ and $X_{h} \subset C^{1}(\bar{\Omega})$ hold. Then the following inclusions hold:

$$
\begin{aligned}
& X_{h} \subset H^{2}(\Omega), \\
& X_{o h}=\left\{v_{h} \in X_{h}: v_{h}=0 \text { on } \Gamma_{0}\right\} \subset V_{2}, \\
& X_{\text {ooh }}=\left\{v_{h} \in X_{h}: v_{h}=\partial_{n} v_{h}=0 \text { on } \Gamma_{0}\right\} \subset V_{1} .
\end{aligned}
$$

Remark 2.6. The choices $V_{h}=X_{o o h}$ for $(B C)_{1}$ and $V_{h}=X_{o h}$ for $(B C)_{2}$ ensure hypotheses (19) to (21) of Theorem 2.3 to be satisfied [7].

Finite elements of class $C^{1}$ are rather complicated and time-consuming, and are not used too often in practical applications. We choose to start our experiments with such elements because the theory described is valid for conforming approximations. In this context, the HCT is one of the simplest $C^{1}$ elements (Fig. 1(a)). The set of degrees of freedom (twelve in total) is given by the values of a function, as well as of its partial derivatives, at the three vertices and by the values of its normal derivatives at the midpoints of the sides. From an internal viewpoint, the HCT element is a composite element: a typical triangle $K \in \mathcal{T}_{h}$ is split into three sub-triangles $K_{i}(i=1,2,3)$, the internal node usually corresponding to the barycenter of $K$ (Fig. 1(b)). A polynomial of degree three is defined on each 
sub-triangle, so that the space $P_{K}$ is given by

$$
P_{K}=\left\{p \in C^{1}(K): p_{\mid K_{i}} \in \mathbb{P}_{3}\left(K_{i}\right), 1 \leqslant i \leqslant 3\right\} .
$$

The condition $p \in C^{1}(K)$ is realized by requiring the continuity of the three polynomial expansions and of their gradients at the barycenter (marked by a black square in Fig. 1(b)), and the continuity of their normal derivatives at the midpoints of the internal sides. Thus, the HCT element is a $C^{1}$ element as a whole, in the sense that a function and its first derivatives are continuous across the edges of any two adjacent elements of $\mathcal{T}_{h}$.

Let us now provisionally focus on the static counterpart of (13), namely,

$$
\forall v \in V, \quad a(w, v)=L(v),
$$

where we have neglected time dependence. Notice that in this case the pivot space $H$ is $L^{2}(\Omega)$. The discrete version of (23) reads

$$
\sum_{j=1}^{I} a\left(\varphi_{i}, \varphi_{j}\right) \xi_{j}=L\left(\varphi_{i}\right), \quad 1 \leqslant i \leqslant I .
$$

The following theorem [7] yields an estimate of the error between $w_{h}$ and $w$ when HCT elements are employed in space discretization for (23).

Theorem 2.7. If the exact solution $w \in V$ of $(23)$ is also in the space $H^{4}(\Omega)$, then there exists a constant $C>0$ independent of $h$ such that

$$
\left\|w-w_{h}\right\|_{H^{2}(\Omega)} \leqslant C h^{2}|w|_{H^{4}(\Omega)} .
$$

Remark 2.8 (Implementation issues). In finite element methods, a quadrature scheme is needed to compute the coefficients $a\left(\varphi_{i}, \varphi_{j}\right)$ and $L\left(\varphi_{i}\right)$, thereby resulting in an approximated bilinear form $a_{h}(\cdot, \cdot)$ and in an approximated linear form $L_{h}(\cdot)$. Generally, integration over a mesh element is performed using a quadrature scheme for which all nodes are situated at the interior of the element. However, in the HCT case, a mesh element features internal interfaces between any two sub-triangles; at these interfaces, the continuity of second partial derivatives is not guaranteed. Hence, one should use a quadrature scheme that avoids nodes on any such interface. A solution is to integrate on each sub-triangle and then sum up the three contributions. Moreover, in order to apply the first Strang lemma [?,7], we need that the bilinear form $a_{h}$ be uniformly $V_{h}$-elliptic, i.e. $\exists \alpha>0: \forall v_{h} \in V_{h}, \quad a_{h}\left(v_{h}, v_{h}\right) \geqslant \alpha\left\|v_{h}\right\|^{2}$. In our situation, if the space $P_{K}$ contains polynomials of degree at most $k$, a sufficient condition for $a_{h}$ to satisfy this property is that the quadrature scheme be exact for polynomials of degree $2 k-4$ at least. This means that on each $K_{i} \subset K$, a quadrature formula exact at least for polynomials of degree two has to be used. 


\section{Numerical simulations}

For what concerns the numerical treatment of problem (1)-(2), we will restrict our attention to an isotropic behavior; hence, the constitutive equation yielding $\mathbf{M}$ is

$$
\mathbf{M}=-D((1-\nu) \nabla \nabla u+\nu \triangle u \mathbf{I}), \quad \text { with } \quad D=\frac{2 e^{3} E}{3\left(1-\nu^{2}\right)}
$$

the flexural rigidity of the plate, $E$ and $\nu$ being respectively Young's modulus and Poisson's ratio of the material.

We perform numerical tests using the software package FreeFEM++ 3.42 (see [13]), in which HCT elements have been implemented along with an adequate quadrature formula for their use. We will consider two situations:

(1) $\Omega=\left\{(x, y) \in \mathbb{R}^{2}: x^{2}+y^{2}<R^{2}\right\}, \Gamma_{0}=\partial \Omega$ and $(B C)_{1}$,

i.e. a circular plate of radius $R$ clamped all over the lateral surface;

(2) $\Omega=(0, a) \times(0, b)$ with $a, b>0, \Gamma_{0}=\partial \Omega$ and $(B C)_{2}$,

i.e. a rectangular plate simply supported all over the lateral surface.

In both situations we assume $\mathbf{m}=\mathbf{0}$, and in order to get coherent results we fix once and for all the following set of data:

$$
\begin{gathered}
R=5 \mathrm{~cm}, a=6 \mathrm{~cm}, b=8 \mathrm{~cm}, e=1 \mathrm{~mm} \\
\rho=5600 \mathrm{~kg} / \mathrm{m}^{3}, E=136 \mathrm{GPa}, \nu=0.3, \\
(\text { i.e. } D=99.63 \mathrm{~N} \cdot \mathrm{m}) .
\end{gathered}
$$

\subsection{Statics}

In each of the following test cases, we consider nested meshes in order to determine the behavior of the relative error in $H^{2}(\Omega)$-norm with respect to the meshsize $h$; of course, mesh refinement is uniform.

\subsubsection{Boundary conditions $(B C)_{1}$}

The problem formulation reads in this case

$$
\begin{cases}D \triangle \triangle w=f_{0} & \text { in } \Omega, \\ w=0, \partial_{n} w=0 & \text { on } \partial \Omega,\end{cases}
$$

with $f_{0}$ a constant; the closed-form solution of (27) is given in this case by

$$
w(x, y)=\frac{f_{0}}{64 D}\left(R^{2}-\left(x^{2}+y^{2}\right)\right)^{2} .
$$


Table 1. Variation of the $H^{2}$-norm of the relative error with the number of finite elements for $w$ given by (28), along with the corresponding number of degrees of freedom.

\begin{tabular}{|c|c|c|c|c|}
\hline Relative error in $\boldsymbol{H}^{\mathbf{2}}$-norm & $0.75 \%$ & $0.2 \%$ & $0.06 \%$ & $0.01 \%$ \\
\hline Number of finite elements & 111 & 444 & 1776 & 7104 \\
\hline Number of degrees of freedom & 386 & 1435 & 5531 & 21715 \\
\hline
\end{tabular}

Table 1 shows that the variation of the $H^{2}$-norm of the error with respect to the number of finite elements (and thus to the meshsize) is in agreement with error estimate (24), i.e. quadratic. A different simulation where the exact solution is non-polynomial, namely, $w(x, y)=\frac{f_{0}}{64 D}\left(R^{2}-\left(x^{2}+y^{2}\right)\right)^{2} \sin (a x)$, gives similar results.

\subsubsection{Boundary conditions $(B C)_{2}$}

The problem formulation is

$$
\begin{cases}D \triangle \triangle w=f_{0} \sin \left(\frac{\pi}{a} x\right) \sin \left(\frac{\pi}{b} y\right) & \text { in } \Omega, \\ w=0, \mathbf{M n} \cdot \mathbf{n}=0 & \text { on } \partial \Omega,\end{cases}
$$

The closed-form solution is given by

$$
w(x, y)=W_{0} \sin \left(\frac{\pi}{a} x\right) \sin \left(\frac{\pi}{b} y\right), \quad W_{0}=\frac{f_{0}}{\pi^{4} D}\left(\frac{1}{a^{2}}+\frac{1}{b^{2}}\right)^{-2} .
$$

The variation of the relative error is shown in Table 2, and once more the convergence is quadratic.

Table 2. Variation of the relative error in $H^{2}$-norm with the number of finite elements for $w$ given by (30), along with the corresponding number of degrees of freedom.

\begin{tabular}{|c|c|c|c|c|}
\hline Relative error in $\boldsymbol{H}^{\mathbf{2}}$-norm & $0.4 \%$ & $0.1 \%$ & $0.03 \%$ & $0.009 \%$ \\
\hline Number of finite elements & 212 & 848 & 3392 & 13568 \\
\hline Number of degrees of freedom & 719 & 2707 & 10499 & 41347 \\
\hline
\end{tabular}

\subsection{3 $L$-shaped clamped plate}

To further test the effectiveness of HCT elements, we also performed a numerical simulation in the case of an $L$-shaped clamped plate, i.e. with boundary conditions $(B C)_{1}$. Table 3 shows the variation of the relative error in $H^{2}$-norm. Since the 


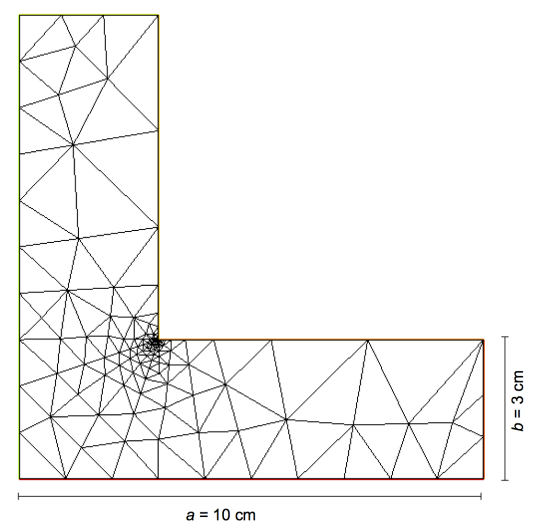

Figure 2. $L$-shaped domain.

closed-form solution is not known in this case, comparison has been made with a numerical solution obtained on a fine adapted mesh, consisting of 137232 elements (meshsize $0.08 \mathrm{~mm}$ ). Computations have been carried out, again, with nested meshes, taking into account the singularity at the inside corner with a suitablyrefined initial mesh. The corvergence rate turns out to be slightly slower than quadratic (1.74). Since the solution has very large variations around the inside corner, adaptive mesh refinement is necessary; however, such numerical aspects are beyond the scope of this paper.

Table 3. Variation of the $H^{2}$-norm of the relative error with the number of finite elements for $w$ given by (28), along with the corresponding number of degrees of freedom.

\begin{tabular}{|c|c|c|c|c|}
\hline Relative error in $\boldsymbol{H}^{\mathbf{2}}$-norm & $24.3 \%$ & $7.3 \%$ & $2.2 \%$ & $0.8 \%$ \\
\hline Number of finite elements & 258 & 1032 & 4128 & 16512 \\
\hline Number of degrees of freedom & 869 & 3283 & 12755 & 50275 \\
\hline
\end{tabular}

\subsection{Dynamics}

In order to test the accuracy of the Newmark midpoint method combined with HCT elements, we consider the time evolution of the transverse displacement $t \mapsto$ $w_{h}\left(x_{0}, y_{0} ; t\right)$, where $\left(x_{0}, y_{0}\right)$ is the center of the plate; we consider nested meshes in the two cases $(B C)_{1}$ and $(B C)_{2}$. The data set is given in (26). We consider an exact solution of the form $w(x, y ; t)=k(x, y) \sin (\beta t)$, where $\beta$ is a constant ${ }^{1}$,

\footnotetext{
${ }^{1}$ We obtained similar results for time dependencies of the form $t^{2}$ and $\arctan (\beta t)$.
} 
and we take into account the evolution of the relative error

$$
\varepsilon_{h}(t)= \begin{cases}\left|\frac{w_{h}\left(x_{0}, y_{0} ; t\right)-w\left(x_{0}, y_{0} ; t\right)}{w\left(x_{0}, y_{0} ; t\right)}\right| & \text { if } t>0, w\left(x_{0}, y_{0} ; t\right) \neq 0 \\ 0 & \text { if } t=0 .\end{cases}
$$

Since convergence occurs only for $0<t \leqslant T$ where $T$ is fixed, we precise in each example the value of $T$.

\subsubsection{Influence of space discretization}

In this section we fix once and for all the time-step to $\Delta t=0.01 \mathrm{~s}$, and we point out the influence of space discretization.

\section{Boundary conditions $(B C)_{1}$}

The function

$$
w(x, y ; t)=\frac{f_{0}}{64 D}\left(R^{2}-\left(x^{2}+y^{2}\right)\right)^{2} \sin (\beta t),
$$

with $f_{0}$ a constant, is solution to the problem

$$
\begin{cases}2 e \rho \ddot{w}-\frac{2 e^{3}}{3} \rho \triangle \ddot{w}+D \triangle \Delta w=f & \text { in } \Omega \times(0, T), \\ w_{0}(x, y)=0, w_{1}(x, y)=\frac{f_{0} \beta}{64 D}\left(R^{2}-\left(x^{2}+y^{2}\right)\right)^{2} & \text { in } \Omega, \\ w=0, \partial_{n} w=0 & \text { on } \partial \Omega,\end{cases}
$$

with

$$
\begin{aligned}
f(x, y ; t)= & -\frac{\beta^{2} f_{0} e \rho}{96 D}\left(3\left(R^{2}-\left(x^{2}+y^{2}\right)\right)^{2}+8 e^{2}\left(R^{2}-2\left(x^{2}+y^{2}\right)\right)\right) \sin (\beta t) \\
& +f_{0} \sin (\beta t) .
\end{aligned}
$$

The evolution of the error given by (31) is shown in Fig. 3(a), and it reflects the expected behavior: the error evolution is attenuated upon refining the mesh.

\section{Boundary conditions $(B C)_{2}$}

The function

$$
w(x, y ; t)=W_{0} \sin \left(\frac{\pi}{a} x\right) \sin \left(\frac{\pi}{b} y\right) \sin (\beta t)
$$


with $W_{0}$ as in (30), is solution to the problem

$$
\begin{cases}2 e \rho \ddot{w}-\frac{2 e^{3}}{3} \rho \Delta \ddot{w}+D \Delta \triangle w=f & \text { in } \Omega \times(0, T), \\ w_{0}(x, y)=0, w_{1}(x, y)=\beta W_{0} \sin \left(\frac{\pi}{a} x\right) \sin \left(\frac{\pi}{b} y\right) & \text { in } \Omega, \\ w=0, \mathbf{M n} \cdot \mathbf{n}=0 & \text { on } \partial \Omega,\end{cases}
$$

with

$$
\begin{aligned}
& f(x, y ; t)= \\
& =-\frac{2 \beta^{2} a^{2} b^{2} f_{0} e\left(b^{2} e^{2} \pi^{2}+a^{2}\left(3 b^{2}+e^{2} \pi^{2}\right)\right)}{3 \pi^{4} D\left(a^{2}+b^{2}\right)^{2}} \rho \sin \left(\frac{\pi}{a} x\right) \sin \left(\frac{\pi}{b} y\right) \sin (\beta t)+ \\
& \quad+f_{0} \sin \left(\frac{\pi}{a} x\right) \sin \left(\frac{\pi}{b} y\right) \sin (\beta t) .
\end{aligned}
$$

The evolution of the relative error given by (31) is shown in Fig. 3(b). Let us remark that the behavior of the error corresponding to the finest mesh (represented by a red line) is in this case almost imperceptible, inasmuch as it is very close to zero. Also, notice that the convergence is in this case remarkably faster than in Fig. 3(a); this can be related to the fact that no approximation error concerning the domain geometry is committed, unlike the case of $(B C)_{1}$, where the domain under consideration is a circle.

\subsubsection{Influence of time discretization}

In this section we consider two different values of the time-step $\Delta t$ (namely, $0.01 \mathrm{~s}$ and $0.05 \mathrm{~s}$ ) in order to point out the influence of this parameter.

In the case of $(B C)_{2}$, when the source term vanishes $(f \equiv 0)$, the closedform solution to the dynamic problem can be obtained by separation of variables. Indeed, one has the Fourier development

$$
w(x, y ; t)=\sum_{m, n \in \mathbb{N}}\left(g_{m n}^{1} \cos \left(\omega_{m n} t\right)+g_{m n}^{2} \sin \left(\omega_{m n} t\right)\right) \sin \left(\frac{m \pi}{a} x\right) \sin \left(\frac{n \pi}{b} y\right),
$$

where

$$
\omega_{m n}=\pi^{2}\left(\frac{m^{2}}{a^{2}}+\frac{n^{2}}{b^{2}}\right) \sqrt{\frac{D}{2 e \rho+\frac{2}{3} \pi^{2} e^{3} \rho\left(\frac{m^{2}}{a^{2}}+\frac{n^{2}}{b^{2}}\right)}},
$$

and coefficients $g_{m n}^{1}$ and $g_{m n}^{2}$ are determined by initial conditions. We consider then the following situation:

$$
w_{0}(x, y) \equiv 0, \quad \text { and } \quad w_{1}(x, y)=\alpha \sin \left(\frac{\pi}{a} x\right) \sin \left(\frac{\pi}{b} y\right),
$$




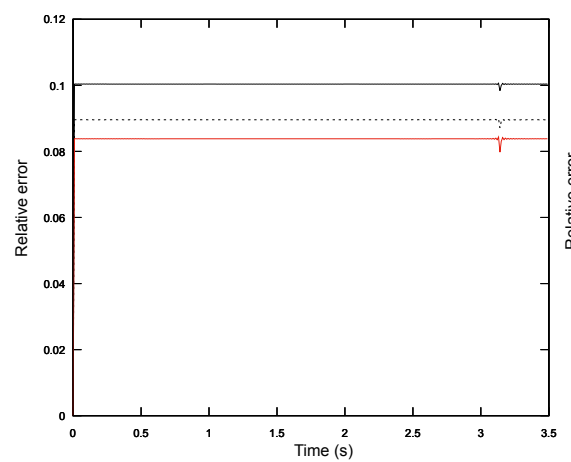

(a)

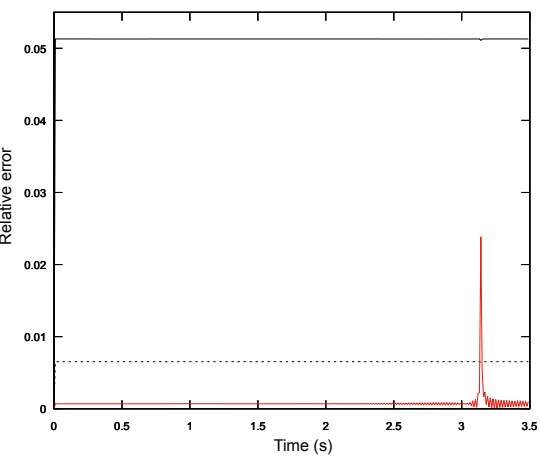

(b)

Figure 3. Evolution of the relative error $t \mapsto \varepsilon_{h}(t)$ with $\left(x_{0}, y_{0}\right)=(0,0)$ for $(B C)_{1}$ (a) (continuous line: 27 elements; dashed line: 108 elements; red line: 432 elements), and with $\left(x_{0}, y_{0}\right)=(a / 2, b / 2)$ for $(B C)_{2}$ (b) (continuous line: 12 elements; dashed line: 48 elements; red line: 192 elements). In both cases, $\beta=1 \mathrm{~s}^{-1}$ and $T=3.5 \mathrm{~s}$. In both cases, all three evolutions become slightly irregular (more remarkably in case (b), with the largest number of elements) when $t$ is close to $\pi$; indeed, $g(\pi)=0$ for $\beta=1 s^{-1}$.

in which case the exact solution is

$$
w(x, y ; t)=\frac{\alpha}{\omega_{11}} \sin \left(\frac{\pi}{a} x\right) \sin \left(\frac{\pi}{b} y\right) \sin \left(\omega_{11} t\right)
$$

Given that the exact solution is $\left(2 \pi / \omega_{11}\right)$-periodic in time, in order to test our numerical method we consider a reasonable value of $\omega_{11}$; say, $\omega_{11}=10 \mathrm{~s}^{-1}$. The evolution of the relative error, given by (31), is shown in Fig. 4(a) for a time-step $\Delta t=0.05 \mathrm{~s}$ and in Fig. 4(b) for a time-step $\Delta t=0.01 \mathrm{~s}$. For $\Delta t=0.05 \mathrm{~s}$, the obtained behavior is unexpected: mesh refinement results in an amplification of the relative error ; decreasing the time-step to $\Delta t=0.01 s$ yields the expected behavior. Indeed, note that the period $\tau$ corresponding to $\omega_{11}=10 \mathrm{~s}^{-1}$ is approximately equal to $0.63 \mathrm{~s}$, so that the ratio $\Delta t / \tau$ is about 0.08 for $\Delta t=0.05 \mathrm{~s}$ and about 0.01 for $\Delta t=0.01 \mathrm{~s}$. The influence of such ratio is well-known in the case of Newmark's midpoint method (see, e.g., [12]). 


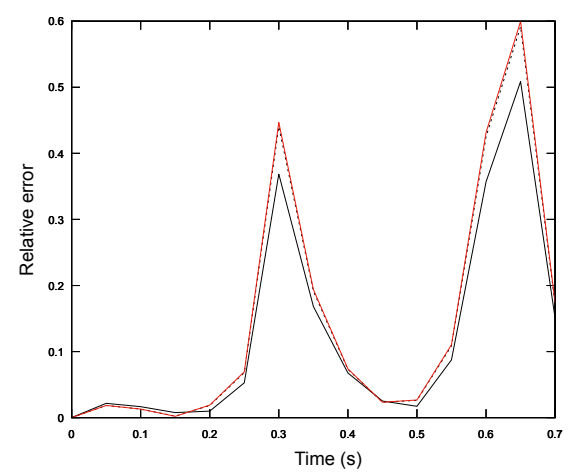

(a)

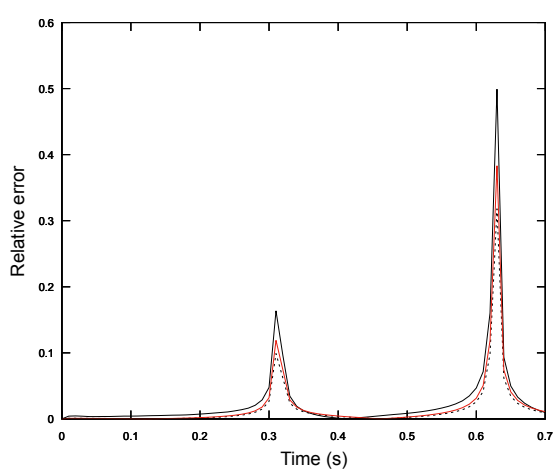

(b)

Figure 4. Evolution of the relative error $t \mapsto \varepsilon_{h}(t)$, with $\left(x_{0}, y_{0}\right)=(a / 2, b / 2)$, for a vanishing initial displacement and a nonzero initial velocity, for a time-step $\Delta t=0.05 s$ (a) and a time-step $\Delta t=0.01 s(\mathrm{~b})$. Continuous line: 28 elements; dashed line: 112 elements; red line: 448 elements. In both cases, $\alpha=1 \mathrm{~cm} / \mathrm{s}$ and $T=0.7 s$.

\section{Concluding remarks}

We have pointed out the importance of our choice of spaces $V$ and $H$, required for the treatment of the rotational inertia term, not only in the proof of the problem's well-posedness, but also in error estimates concerning our numerical method. Our simulations, performed using FreeFEM++ 3.42 show that the presence of the rotational inertia term does not affect the efficiency of the Newmark time discretization method combined with conforming finite elements such as HCT elements. We have tested the implementation of such elements in three cases: clamped circular plate, simply supported rectangular plate and $L$-shaped clamped plate. In all these cases, our numerical experiments validate the convergence rate of the error predicted by the theory. As is well-known, HCT elements are computationally expensive; it would then be of interest to use nonconforming space discretization methods (mixed or hybrid) [4,17], such as HHO methods [8,9]. The application of such methods to plate problems seems particularly interesting and will be carried out in a forthcoming work.

Acknowledgments. We thank Frédéric Hecht for his support and collaboration. 


\section{Bibliography}

[1] G. Allaire, Numerical Analysis and Optimization, Oxford University Press (2007).

[2] G. A. Baker, Error estimates for finite element methods for second order hyperbolic equations, SIAM J. Numer. Anal. 13 (1976), 564-576.

[3] J.-L. Batoz, K.-J. Bathe, L.-W. Ho, A study of three-node triangular plate bending elements, Internat. J. Numer. Methods Engrg. 12 (1980), 1771-1812.

[4] D. Boffi, F. Brezzi, M. Fortin, Mixed finite element methods and applications, Springer-Verlag (2013).

[5] F. Brezzi, M. Fortin, Mixed and hybrid finite element methods, Springer Science \& Business Media (2012).

[6] F. Bonaldi, G. Geymonat, F. Krasucki, M. Serpilli, An asymptotic plate model for magneto-electro-thermo-elastic sensors and actuators, Math. Mech. Solids (2015), published online. DOI: 10.1177/1081286515612885.

[7] P. G. Ciarlet, The Finite Element Method for Elliptic Problems, SIAM (2002).

[8] D. A. Di Pietro, A. Ern, S. Lemaire, An Arbitrary-Order and Compact-Stencil Discretization of Diffusion on General Meshes Based on Local Reconstruction Operators, Comput. Methods Appl. Math. 14 (2014), 461-472.

[9] D. A. Di Pietro, A. Ern, A hybrid high-order locking-free method for linear elasticity on general meshes, Comput. Methods Appl. Mech. Engrg. 283 (2015), 1-21.

[10] R. Dautray, J.-L. Lions, Mathematical Analysis and Numerical Methods for Science and Technology, Volume 5 - Evolution Problems I, Springer-Verlag (2000).

[11] T. Dupont, $L^{2}$-Estimates for Galerkin Methods for Second Order Hyperbolic Equations, SIAM J. Numer. Anal. 10 (1973), 880-889.

[12] M. Géradin, D. J. Rixen, Mechanical Vibrations: Theory and Application to Structural Dynamics, John Wiley \& Sons (2015).

[13] F. Hecht, New development in FreeFem++, J. Numer. Math. 20 (2012), 251-265.

[14] T. J. R. Hughes, The finite element method: linear static and dynamic finite element analysis, Courier Corporation (2012).

[15] J.-L. Lions, E. Magenes, Non-Homogeneous Boundary Value Problems and Applications, Springer-Verlag (1972).

[16] R. D. Mindlin, Influence of rotatory inertia and shear on flexural motions of isotropic, elastic plates, Journal of Applied Mechanics 18 (1951), 31-38.

[17] A. Quarteroni, A. Valli, Numerical Approximation of Partial Differential Equations, Springer-Verlag (2008).

[18] A. Raoult, Construction d'un modèle d'évolution de plaques avec terme d'inertie de rotation, Ann. Mat. Pura Appl. CXXXIX (1985), 361-400 (French). 
[19] P. A. Raviart, J. M. Thomas, Introduction à l'analyse numérique des équations aux dérivées partielles, Masson (1983).

[20] J. W. S. Baron Rayleigh, The Theory of Sound, 2nd ed., 1894, reprinted Dover edition (1945).

[21] G. Strang, G. J. Fix, An Analysis of the Finite Element Method, Prentice-Hall (1973).

\section{Author information}

Francesco Bonaldi, Institut Montpelliérain Alexander Grothendieck, UMR-CNRS 5149, Université de Montpellier, Place Eugène Bataillon, 34095 Montpellier Cedex 5, France.

E-mail: francesco.bonaldi@umontpellier.fr

Giuseppe Geymonat, Laboratoire de Mécanique des Solides, UMR-CNRS 7649,

École Polytechnique, CNRS, Université Paris-Saclay,

Route de Saclay, 91128 Palaiseau Cedex, France.

E-mail: giuseppe.geymonat@lms.polytechnique.fr

Françoise Krasucki, Institut Montpelliérain Alexander Grothendieck, UMR-CNRS 5149, Université de Montpellier, Place Eugène Bataillon, 34095 Montpellier Cedex 5, France. E-mail: francoise.krasucki@umontpellier.fr

Marina Vidrascu, REO project-team, Inria de Paris, 2 rue Simone Iff Voie DQ12, 75012 Paris, France. Laboratoire Jacques-Louis Lions, UPMC Univ. Paris 6, Sorbonne Universités, 4 Place Jussieu, 75252 Paris Cedex 5, France.

E-mail: marina.vidrascu@inria.fr 